\title{
Temporary Epicardial Pacing Wire Migration into the Right Heart, 10 Months after Coronary Artery Bypass Surgery
}

\author{
Sungjoon Park, MD, ${ }^{1}$ Jaehoon Lee, MD, ${ }^{1}$ Young-Sup Byun, MD,${ }^{2}$ In Hyun Jung, MD, ${ }^{2}$ Euysuk Chung, MD $^{1}$ \\ ${ }^{1}$ Department of Cardiothoracic Surgery, School of Medicine, Inje University, Sanggye Paik Hospital, Seoul, South Korea; \\ ${ }^{2}$ Department of Cardiology, School of Medicine, Inje University, Sanggye Paik Hospital, Seoul, South Korea
}

\section{ABSTRACT}

Migrations of retained temporary epicardial pacing wires (TEPWs) are rare and critical complications of cardiac surgery. A 73-year-old man who had received coronary artery bypass graft (CABG) with retained TEPW 10 months previously visited the outpatient clinic. In routine echocardiography, we observed an artificial structure in the right heart. We performed computed tomography (CT), identified TEPW in the right heart, confirmed the TEPW migration process by comparing it with previous $\mathrm{CTs}$, and removed it via catheter intervention. We report this rare case because we identified TEPW in the heart, determined its migration process, and removed it without complications.

\section{INTRODUCTION}

Temporary epicardial pacing wires (TEPWs) are widely used at the last stage of cardiac surgery in case of postoperative arrhythmia, and removal is carried out before discharge [Khorsandi 2012]. The overall incidence of major complications related to TEPW removal is low, but when complications occur, they can be significant (bleeding, cardiac tamponade, or hemopericardium) [Del Nido 1989]. Occasionally, therefore, the wires are cut just at the skin level in case of bleeding tendencies, and resistance is encountered [Carroll 1998]. Retained TEPWs can lead to early and delayed complications, however, such as irritation, pain, infection, and distant migration. Distant migration of retained TEPWs is a rare but serious complication [Shaikhrezai 2012]. We found that a retained TEPW had migrated from the pericardial space into the right heart 10 months after coronary artery bypass graft (CABG) surgery.

\section{CASE PRESENTATION}

A 73 year-old-male, who underwent coronary CABG (left internal thoracic artery to left anterior descending coronary

Received October 29, 2019; accepted Fanuary 24, 2020.

Correspondence: Euysuk Chung, Department of Cardiothoracic Surgery, School of Medicine, Inje University, Sanggye Paik Hospital, 24-1, Sanggye-ro 10-gil, Nowon-gu, Seoul, Republic of Korea; 82001086626890 (e-mail: kustricha@ banmail.net). artery, right internal thoracic artery to obtuse marginal artery) 10 months previously because of unstable angina and 3-vessel disease, visited the outpatient clinic for a routine follow-up and echocardiography. The echocardiography results showed that cardiac function had improved, and there were no myocardial wall motion abnormalities. However, we observed an artificial structure moving in the right ventricle (RV) and the right atrium (RA). One end of the artificial structure was embedded in the RV septum, and the other end was in the RV free wall; the opposite U-shaped middle part was observed in the RA (Figures 1a and 1b).

We reviewed the patient's old admission data. During the CABG, we had applied the TEPW (Bipolar VF series, Medical Concepts Europe, Gemert, Netherland) on the anterior wall of the RV. Before being discharged, the patient had been taking antiplatelet agents (aspirin, clopidogrel), and because of the resulting bleeding tendency, we cut the TEPW at the skin level. After the echocardiography 10 months later, we suspected distant migration of the remaining TEPW and reviewed the old echocardiography and computed tomography (CT) data.

There were no foreign bodies in the heart at the immediate postoperative echocardiography. However, on the 1-month cardiac CT, we found a TEPW distal tip embedded 1 to $2 \mathrm{~mm}$ inside the RV myocardium; there was no foreign body in the RA or RV (Figures 2a, 2b, and 2c). We performed a cardiac CT again and found that the TEPW had moved entirely into the right heart. Both ends of the TEPW were in the RV septum and apex, and the U-shaped middle portion of the TEPW had passed through the tricuspid valve and into the RA (Figures 2c, 2d, and 2e).

We planned to remove the TEPW via catheter. Under fluoroscopy, we observed that the U-shaped TEPW was moving with the heartbeat, with both ends embedded in the
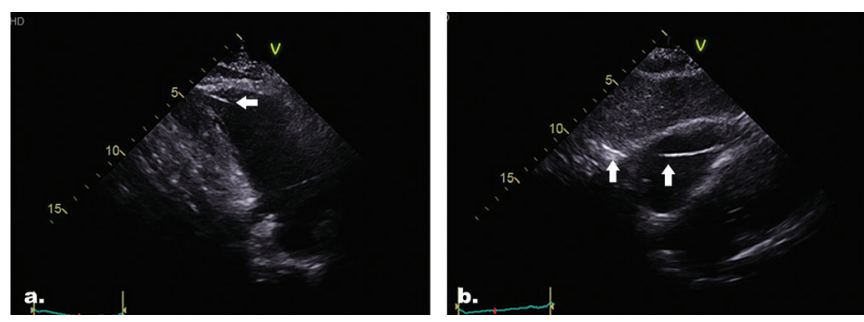

Figure 1. Postoperative 10-month echocardiography. a, TEPW in the right ventricle. b, TEPW in the right atrium. 

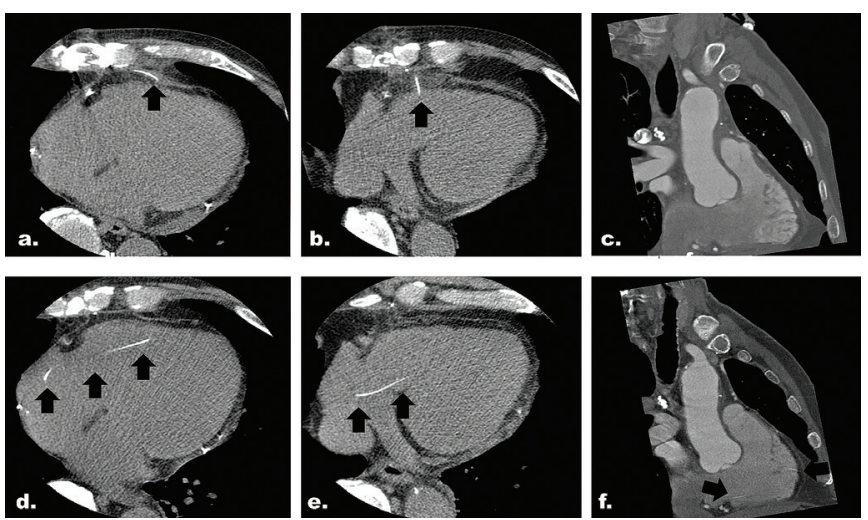

Figure 2. Cardiac computed tomography 1 month (a, b, c) and 10 months ( $d$, e, f) after surgery. a, TEPW is in the pericardial space. b, TEPW tip is embedded inside the right ventricle myocardium. c, TEPW is not visualized in the right heart. $d$ and e, TEPW is in the RA and RV. $f$, Both tips of the TEPW are trapped in the RA and ventricle wall (black arrow).

RV muscle. We inserted the curved catheter (Performa ${ }^{\circledR}$ Judkins Right Catheters 4.0, Merit Medical, South Jordan, UT), hooked it to the middle of the TEPW, and pulled the wire to move one of its ends in the RV muscle to the infra vena cava. After that, using the snare (Multi-Snare ${ }^{\circledR}$ Micro Sets $4 \mathrm{~mm}$, PFM Medical, Cologne, Germany), the TEPW was captured and removed without any significant resistance (Figures 3a, 3b, 3c, and 3d). The patient was discharged the day after this procedure without further complications.

\section{DISCUSSION}

TEPWs are routinely used at the last stage of cardiac surgery because they are helpful in the postoperative management of low cardiac output, arrhythmia, and conduction abnormalities [Khorsandi 2012; Del Nido 1989]. We commonly remove the wire by gentle traction before discharge, and the overall incidence of TEPW-related complications is low. In a small number of patients $(0.4 \%)$, however, TEPW removal can result in critical complications, such as coronary graft and myocardium injury, bleeding, and mortality [Khorsandi 2012; Del Nido 1989; Carroll 1998; Elmistekawy 2019]. Therefore, in case of bleeding tendencies resulting from antiplatelet and warfarin medications, TEPWs are cut just at the skin level and retained [Carroll 1998; Shaikrezai 2012]. Retained TEPWs may cause early complications such as irritation, pain, and erosion, and there have been reports of delayed complications associated with retained TEPWs, such as abscess formation, infection, and distant migration [Shaikrezai 2012; Elmistekawy 2019].

The migration of retained TEPWs is an extremely rare but serious complication [Shaikrezai 2012; Elmistekawy 2019]. Retained wires can migrate to other structures with no clear mechanism. It is possible that they migrate into the chest structure with chest and diaphragm movement. In some reports, abnormal migration of TEPWs was found in places other than
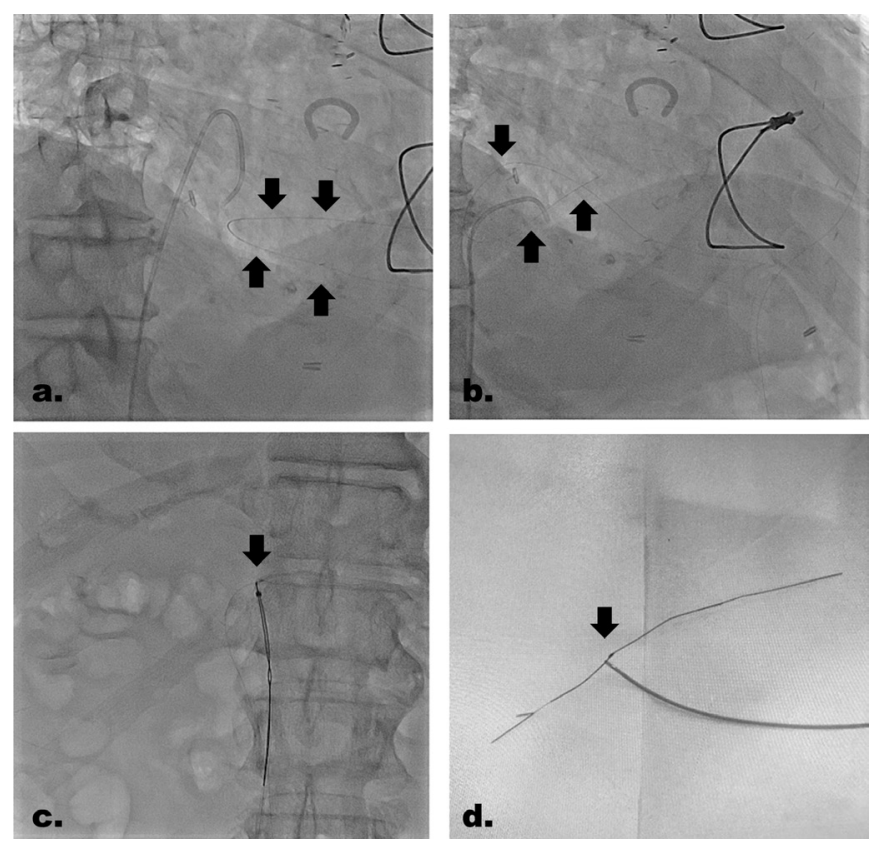

Figure 3. Catheter intervention, under fluoroscopy. a, U-shaped TEPW in RA. b, Hooking TEPW with curved catheter. c, TEPW captured with endovascular snare. d, TEPW removed with endovascular snare.

the circulation system [Kondo 2014; Ghazali 2018]. Another potential mechanism is direct penetration to the myocardium, migration with cardiac pulsation into the cardiac structures, and flow in the circulation system [Gayanilo 2014; Sugiyama 2018; Mukaihara 2015]. This mechanism could be confirmed by observing changes in the CT of our case. In just 10 months, the TEPW, which was initially located primarily in the pericardium and partially embedded in the epicardium, had migrated entirely into the right heart. However, the TEPW did not travel to the pulmonary artery with blood flow, but rather moved to the RA against blood flow.

As in our case, if the retained TEPW is found in the circulatory system, it can be removed by catheter intervention without complications [Gayanilo 2014; Sugiyama 2018]. However, some cases of retained TEPWs in the circulation system or other structures could require surgical removal [Kondo 2014; Ghazali 2018; Mukaihara 2015].

The optimal site to reduce the migration of retained TEPWs is not clear. The RV anterior wall, the most common site for TEPW insertion, has a high rate of dislodgement $(27.8 \%)$, due to the retrosternal chest tube and heartbeat, which may increase the possibility of TEPW migration [Hurle 2002]. Conversely, considering the high conductivity and the low TEPW dislodgment rate, some authors recommend the diaphragmatic ventricle wall to be the optimal site for TEPW insertion [Hurle 2002]; however, in that location, TEPWs may also be affected by the movement of the diaphragm.

We have reported this case because we found the TEPW in the right heart and removed it via catheter without complications. We confirmed the TEPW's 10 -month migration process 
and the mechanism from the pericardial space and epicardium into the RV and RA through well-documented CT images.

\section{REFERENCES}

Carroll KC, Reeves LM, Andersen G, et al. Risks associated with removal of ventricular epicardial pacing wires after cardiac surgery. Am J Crit Care 1998;7:444-449.

Del Nido P, Goldman BS. Temporary epicardial pacing after open heart surgery: Complications and prevention. J Cardiac Surg 1989;4:99-103.

Elmistekawy E. Safety of temporary pacemaker wires. Asian Cardiovasc Thorac Ann 2019;27:341-346.

Gayanilo MA, Rosenkranz ER, Sandhu SK, Kardon RE. Transmyocardial migration of a temporary epicardial pacing wire: a pediatric case report. World J Pediatr Congen Heart Surg 2014;5:315-317.

Ghazali W, Awagi K, AlZahrani G, Ashkar L, AlGaithy Z. An unusual cause of a breast mass in a 13 -year-old girl: A case report. J Med Case Rep 2018;12:236.
Hurle A, Gomez-Plana J, Sanchez J, et al. Optimal location for temporary epicardial pacing leads following open heart surgery. PACE 2002;25:1049-1052.

Khorsandi M, Muhammad I, Shaikhrezai K, Pessotto R. Is it worth placing ventricular pacing wires in all patients post-coronary artery bypass grafting? Interact Cardiovasc Thorac Surg 2012;15:489-493.

Kondo T, Hirota M, Hoshino J, et al. Temporary epicardial pacing wire migrating to and exiting from the jaw. Ann Thorac Surg 2014;98:2221-2223.

Mukaihara K, Yotsumoto G, Matsumoto K, Imoto Y. Migration of a retained temporary epicardial pacing wire into an abdominal aortic aneurysm. Eur J Cardio-Thorac Surg 2015;48:169-170.

Shaikhrezai K, Khorsandi M, Patronis M, Prasad S. Is it safe to cut pacing wires flush with the skin instead of removing them? Interact Cardiovasc Thorac Surg 2012;15:1047-1051.

Sugiyama K, Koizumi N, Nishibe T, Ogino H. Catheter intervention to treat migrated temporary epicardial pacing wire into the left side of the heart. Interact Cardiovasc Thorac Surg. 2018;27:142-144. 